\title{
A Note on the Numerical Evaluation of Thermal Radiation Characteristics of Diffuse Cylindrical and Conical Cavities
}

\author{
B. A. Peavy \\ Institute for Applied Technology, National Bureau of Standards, Washington, D.C. \\ (January 14, 1966)

\begin{abstract}
Presented are methods that avoid the need to employ an extrapolation technique in the region of the critical points for evaluation of the apparent emmissivity of diffuse cylindrical and conical cavities. The methods involve appropriate substitutions in the integrands of integral equations that are used in analytical solutions for determining the thermal radiation characteristics of diffuse and conical cavities. Equations for either isothermal or nonisothermal surface temperature conditions are provided in a direct form for computations. Numerical results are presented for a general linear temperature distribution along the length of a cylindrical cavity. The method is equally applicable
\end{abstract} \\ for the solution of other problems in integral equations where discontinuities are encountered.
}

Key Words: Conical, cylindrical, emissivity, nonisothermal.

\section{Introduction}

Analytical formulations have been derived $[1,2,3]^{1}$ for determining the radiant interchange in finite length cylindrical cavities, and conical cavities whose bounding surfaces radiate in a gray diffuse manner. These solutions are in the form of integral equations where, unfortunately, most of the integrands exhibit discontinuities with finite limits at critical points, such as the corner of the cylindrical cavity and the apex of the conical cavity. Numerical solutions of these equations in the region of the discontinuities have been previously evaluated by extrapolation techniques. Also, to a lesser degree of difficulty, some of the integrands exhibit slope discontinuities, which for accurate numerical results may involve intricate numerical integration procedures.

The purpose of this paper is to present methods for numerical evaluation of the apparent emissivity in these cavities that avoid use of an extrapolation technique in the region of the critical points. For the main part, the methods involve appropriate substitutions which make the integrands go to zero at the critical points and at the points of slope discontinuity. Impetus for this investigation has been the numerical evaluation of thermal radiation characteristics for parameters not included in the scope of references 1 and 2. A particular need has been the determination of the thermal characteristics for low values of the surface emissivity of shallow cylindrical cavities investigated by Kelly and Moore [4].
Also presented is a system of integral equations for determining the thermal characteristics of diffuse cylindrical and conical cavities whose surfaces are nonisothermal. A few selected numerical results are included for the cylindrical cavity with an arbitrary linear temperature variation over its length.

\section{Cylindrical Cavity}

Analytical formulations for the apparent emissivity of the surfaces of cylindrical enclosures have been derived by Sparrow, Albers, and Eckert [1]. Using the same nomenclature, their eqs (8) and (9) are presented here in a different form.

$$
\begin{aligned}
\epsilon_{a}\left(x_{0}\right)=\epsilon+(1-\epsilon) & \int_{0}^{L / d} \epsilon_{a}(x) K_{1}\left(x_{0}, x\right) d x \\
& +\frac{1-\epsilon}{4}\left(\frac{L}{d}-x_{0}\right) \int_{0}^{1} \epsilon_{a}(r) K_{2}\left(x_{0}, r\right) r d r \\
\epsilon_{a}(r)=\epsilon+ & \frac{1-\epsilon}{2} \int_{0}^{L / d} \epsilon_{a}(x) K_{2}(x, r)\left(\frac{L}{d}-x\right) d x
\end{aligned}
$$

where $K_{1}\left(x_{0}, x\right)=1-\frac{\left|x_{0}-x\right|}{2} \frac{2\left(x_{0}-x\right)^{2}+3}{\left\{\left(x_{0}-x\right)^{2}+1\right\}^{3 / 2}}$

$$
\begin{aligned}
K_{2}(x, r) & =\frac{(z+w)}{\left\{z^{2}+(1-2 w) z+w^{2}\right\}^{3 / 2}} \\
z & =\left(\frac{L}{d}-x\right)^{2} \quad \text { and } \quad w=\frac{1-r^{2}}{4}
\end{aligned}
$$


and $\epsilon_{a}$ is the apparent emissivity of a surface location; $\epsilon_{a}\left(x_{0}\right)$ and $\epsilon_{a}(r)$ represent values on the curved surface and flat surface, respectively, of the cylindrical cavity. The physical system is described in [1]. $L$ is the length of the cavity, $d$ is the diameter of the cavity, $x$ is a ratio of the distance measured along the cavity wall from the opening to the diameter, and $r$ is the ratio of the radius of a position on the bottom of the cavity to the cavity radius, $R$.

Initial attempts by the author at obtaining numerical solutions of (1) and (2) were frustrated by the erratic behavior of the kernels $K_{2}\left(x_{0}, r\right)$ and $K_{2}(x, r)$ as $r$ approaches unity and $x$ and $x_{0}$ approach $L / d$ (the corner of the system). Visual inspection of these kernels and integrals involved indicates an indeterminate form at these limits. In correspondence with $\mathrm{L}$. U. Albers (coauthor of [1]), it was disclosed that an extrapolation technique was employed in the region of the corner, and that the results were quite insensitive to wide variations in types of extrapolation. Attempts at extrapolation by the author proved to be inconclusive. Indications were that the second differences were quite large in this region, showing that linear extrapolation may be inaccurate.

The suggested method for obtaining solutions is to make the integrand zero at the corner and make contributions to the results negligibly small in the region of the corner. This may be performed by making the substitutions

$$
\begin{aligned}
& \epsilon_{a}(x)=A_{0}+A_{1} z+\phi(x) \\
& \epsilon_{a}(r)=B_{0}+B_{1} w+\psi(r)
\end{aligned}
$$

in the integrals of (1) and (2). $A_{0}, A_{1}, B_{0}$, and $B_{1}$ are chosen such that $\phi(L / d)=\phi(0)=0$ and $\psi(1)=\psi(0)=0$. Substitution of the first two terms of (3) and (4) in the appropriate integrals of (2) and (1), and by $r d r=-2 d w$, and $\left(\frac{L}{d}-x\right) d x=-d z / 2$ gives

$$
\begin{aligned}
& I_{1}=2 z_{0}^{1 / 2} \int_{0}^{1 / 4} \frac{\left(B_{0}+B_{1} w\right)\left(z_{0}+w\right) d w}{\left\{w^{2}-2 z_{0} w+z_{0}+z_{0}^{2}\right\}^{3 / 2}} \\
& I_{2}=\frac{1}{2} \int_{0}^{(L / d)^{2}} \frac{\left(A_{0}+A_{1} z\right)(z+w) d z}{\left\{z^{2}+(1-2 w) z+w^{2}\right\}^{3 / 2}} .
\end{aligned}
$$

From a table of integrals

$$
\begin{gathered}
I_{1}=2\left[\frac{\left(2 z_{0}+1\right) B_{0}+2 z_{0}\left(z_{0}+1\right) B_{1}}{\left(z_{0}+1\right)^{1 / 2}}\right. \\
\quad-z_{0}^{1 / 2}\left\{2 B_{0}+\left(1+2 z_{0}\right) B_{1}\right\} \\
\left.\quad-z_{0}^{1 / 2} B_{1} \ln \left[2\left\{\left(z_{0}+1\right)^{1 / 2}-z_{0}\right\}\right]\right]
\end{gathered}
$$

$$
\begin{aligned}
& I_{2}=A_{0}+A_{1} w+\frac{\left[(L / d)^{2}-w\right]\left[A_{0}+A_{1}+A_{1} w\right]-A_{1}(L / d)^{2}}{\left[\left\{(L / d)^{2}-w\right\}^{2}+(L / d)^{2}\right]^{1 / 2}} \\
& +A_{1} / 2 \ln \left[2\left\{\left[(L / d)^{2}-w\right]^{2}+(L / d)^{2}\right\}^{1 / 2}+2(L / d)^{2}+1-2 w\right]
\end{aligned}
$$

The kernel $K_{1}\left(x_{0}, x\right)$ has a discontinuous derivative at $x=x_{0}$. This did not seem to have an appreciable effect on the accuracy of numerical integration by Simpson's rule if the subdivision of $L / d$ was sufficiently small. The smaller the increment, the longer becomes the time needed to compute apparent emissivities, and it is therefore expedient to use as large an increment as possible. This can be done by making the integrand equal to zero at $x=x_{0}$, and quite small in the vicinity of $x_{0}$, or by the substitution

$$
\epsilon_{a}(x)=\epsilon_{a}\left(x_{0}\right)+\lambda(x)
$$

in the first integral of eq (1). Letting

$$
I_{3}=\epsilon_{a}\left(x_{0}\right)\left\{\int_{0}^{x_{0}} K_{1}\left(x_{0}, x\right) d x+\int_{x_{0}}^{L / d} K_{1}\left(x_{0}, x\right) d x\right\}
$$

and performing the indicated integration, we obtain

$$
I_{3}=\epsilon_{a}\left(x_{0}\right)\left[\frac{L}{d}-1-\frac{2 x_{0}^{2}+1}{2\left(x_{0}^{2}+1\right)^{1 / 2}}-\frac{2\left(\frac{L}{d}-x_{0}\right)^{2}+1}{2\left\{\left(\frac{L}{d}-x_{0}\right)+1\right\}^{1 / 2}}\right] .
$$

With the substitution of (3), (4), and (7), eqs (1) and (2) become

$$
\begin{aligned}
\epsilon_{a}\left(x_{0}\right) & =\epsilon+(1-\epsilon)\left[I_{3}+\int_{0}^{L / d} \lambda(x) K_{1}\left(x_{0}, x\right) d x\right] \\
+ & \frac{(1-\epsilon)}{4}\left\{I_{1}+\left(\frac{L}{d}-x_{0}\right) \int_{0}^{1} \psi(r) K_{2}\left(x_{0}, r\right) r d r\right\} \\
\epsilon_{a}(r) & =\epsilon+\frac{(1-\epsilon)}{2}\left\{I_{2}+\int_{0}^{L / d} \varphi(x) K_{2}(x, r)\left(\frac{L}{d}-x\right) d x\right\} .
\end{aligned}
$$

Numerical solutions were obtained from (la) and (2a) by the process of iteration, where all numerical integrations were performed by Simpson's rule and employed an increment equal to $1 / 64$ of the difference of the limits of integration. This increment was proved adequate by comparison with results obtained using an increment of $1 / 128$. For a fixed value of $L / d$, the kernel functions were computed only one time for all variations in the surface emissivity, $\epsilon . K_{1}\left(x_{0}, x\right)$ was computed in a one-dimensional array where proper indexing gave translation about a given value of $x_{0}$. $K_{2}(x, r)$ was computed in two-dimensional array.

Initial values of $\epsilon_{a}(x)$ were determined from rather crude polynomial approximations. Placing the values 


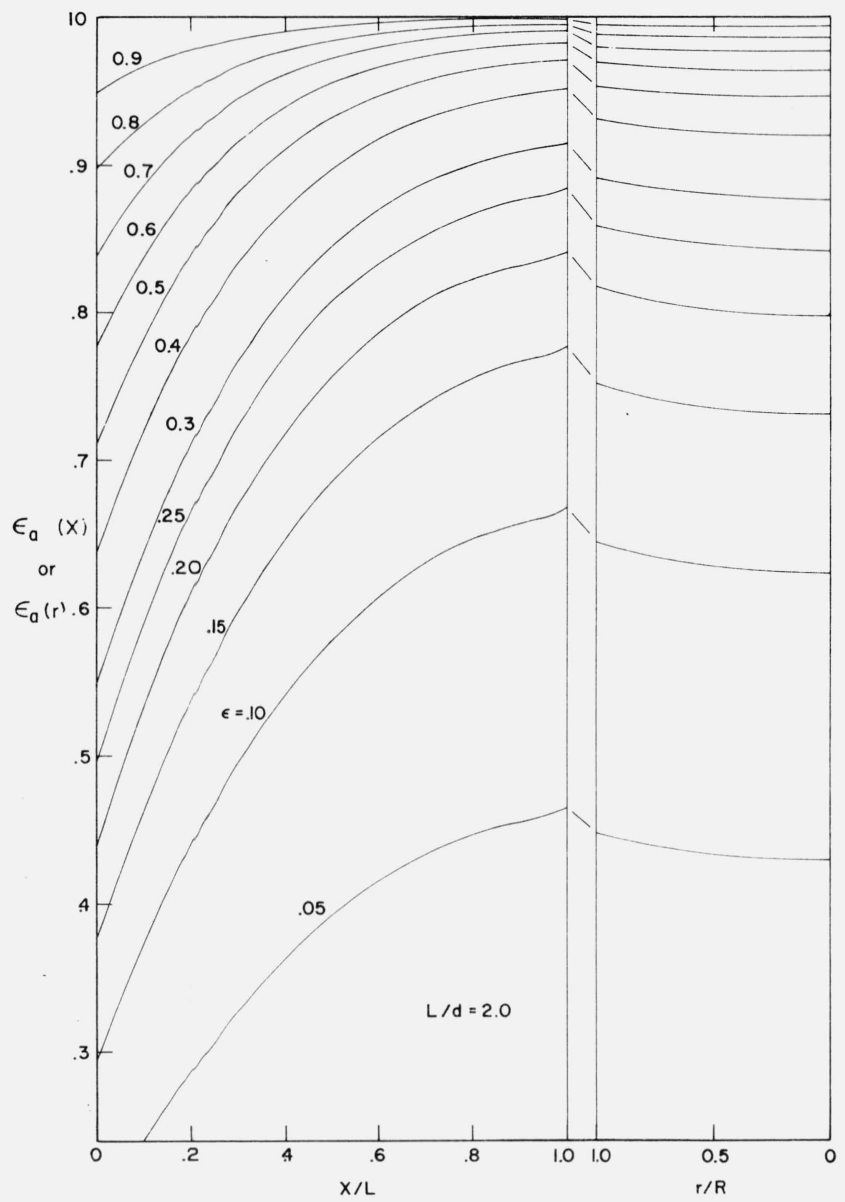

Figure 1. Apparent emissivity in a cylindrical cavity, $\mathrm{L} / \mathrm{d}=2$.

for $\epsilon_{a}(x)$ in (2a), gave values for $\epsilon_{a}(r)$, which with the values of $\epsilon_{a}(x)$ placed in (1a) gave new values for $\epsilon_{a}(x)$. This process was repeated using the new values in (2a). The number of iterations ( $n$ ) necessary for convergence was set by the criterion

$$
\left|\frac{\epsilon_{a}(x=0)_{n}-\epsilon_{a}(x=0)_{n-1}}{\epsilon_{a}(x=0)_{n}}\right| \leqslant 0.0005
$$

which was usually satisfied for $n=4$ or less. A typical time to compute 16 cases involving various values of $L / d$ and $\epsilon$ was about $2 \mathrm{~min}$ for an IBM 7094 digital computer.

Figures 1, 2, 3, and 4 give values for apparent emissivity versus dimension ratios for $L / d=2,1,0.5$, and 0.25 , respectively, and for various values of the surface emissivity, $\epsilon$. Where the same parameters occur, there is agreement with values given in table 1 of reference [1] to within 0.1 percent. Figure 5 is a plot of $\epsilon_{a}(r=0)$ versus $\epsilon$ for $L / d=2,1,0.5$, and 0.25 . Figure 6 is a plot of the ratio of the overall emissivity of the cavity to the surface emissivity $\left(Q / \sigma \in \pi R^{2} T^{4}\right)$ versus $\epsilon$ for $L / d=2,1,0.5$, and 0.25 . The ratio was computed by numerical integration of eq (1lb) of [1].

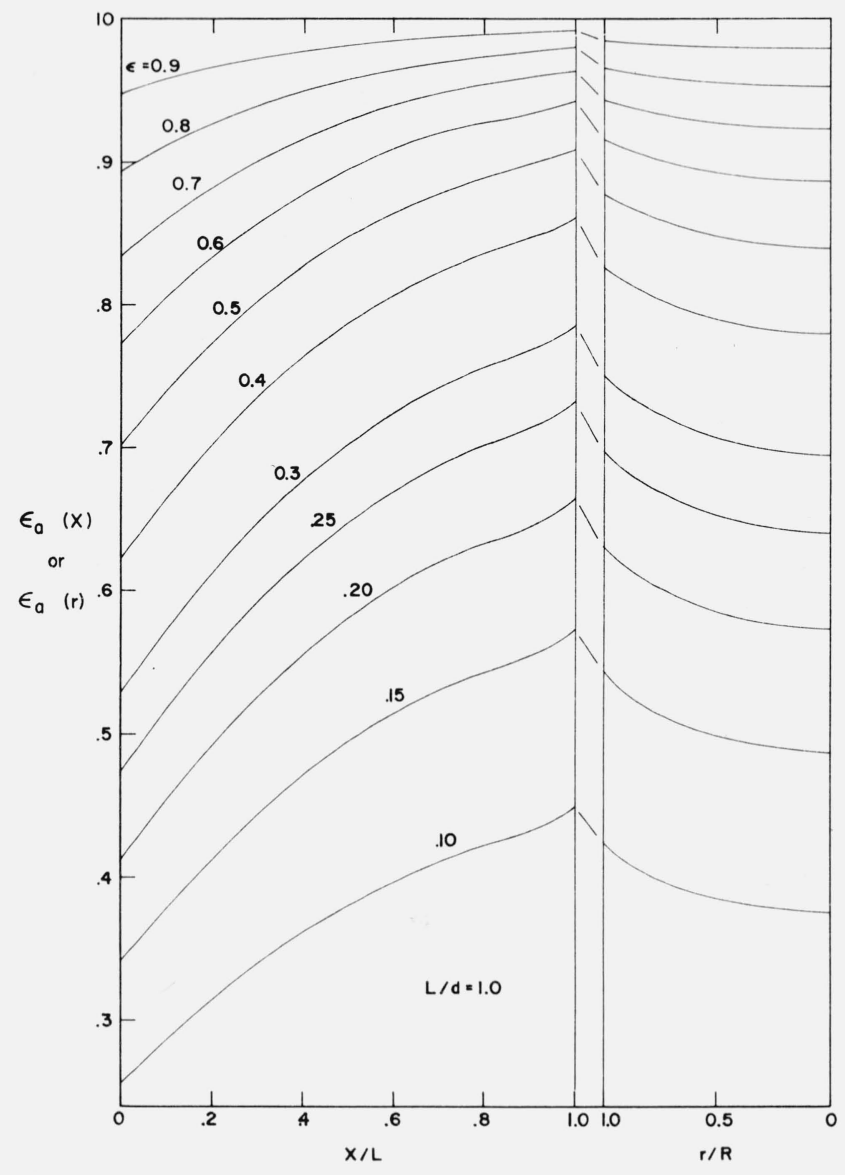

Figure 2. Apparent emissivity in a cylindrical cavity, $\mathrm{L} / \mathrm{d}=1$.

TABLE 1

Values of $\epsilon_{a}(r=0)$ and $\epsilon_{a}(r=1)$

\begin{tabular}{c|rr|rr|rr|rr}
\hline$\epsilon$ & \multicolumn{2}{|c|}{$L / d=2$} & \multicolumn{2}{|c|}{1.0} & \multicolumn{2}{c|}{0.5} & \multicolumn{2}{|c}{0.25} \\
\hline .9 & 0.9936 & 0.9944 & 0.9785 & 0.9843 & 0.9482 & 0.9707 & 0.9191 & 0.9600 \\
.8 & .9860 & .9877 & .9532 & .9659 & .8924 & .9371 & .8361 & .9149 \\
.7 & .9764 & .9795 & .9231 & .9435 & .8314 & .8977 & .7506 & .8633 \\
.6 & .9637 & .9686 & .8862 & .9153 & .7640 & .8504 & .6619 & .8030 \\
.5 & .9460 & .9534 & .8396 & .8778 & .6879 & .7916 & .5694 & .7316 \\
.4 & .9196 & .9302 & .7789 & .8264 & .6005 & .7168 & .4719 & .6452 \\
.3 & .8766 & .8913 & .6951 & .7504 & .4976 & .6185 & .3684 & .5388 \\
.25 & .8442 & .8612 & .6401 & .6980 & .4383 & .5568 & .3138 & .4758 \\
.2 & .7986 & .8179 & .5728 & .6314 & .3724 & .4841 & .2570 & .4046 \\
.15 & .7313 & .7526 & .4874 & .5438 & .2982 & .3973 & .1977 & .3237 \\
.1 & .6238 & .6457 & .3756 & .4246 & .2136 & .2919 & .1354 & .2311 \\
.05 & .4305 & .4488 & .2228 & .2554 & .1156 & .1624 & .0697 & .1242 \\
\hline
\end{tabular}

\begin{tabular}{c|l|l|l|l}
\multicolumn{6}{c}{ Values of $Q /\left(\sigma \in \pi R^{2} T^{4}\right)$} \\
\hline .9 & 1.0829 & 1.0801 & 1.0688 & 1.0483 \\
.8 & 1.1829 & 1.1755 & 1.1482 & 1.1025 \\
.7 & 1.3060 & 1.2908 & 1.2410 & 1.1637 \\
.6 & 1.4620 & 1.4330 & 1.3507 & 1.2337 \\
.5 & 1.6662 & 1.6156 & 1.4844 & 1.3138 \\
.4 & 1.9482 & 1.8540 & 1.6480 & 1.4070 \\
.3 & 2.3670 & 2.1877 & 1.8543 & 1.5163 \\
.25 & 2.6609 & 2.4072 & 1.9788 & 1.5784 \\
.2 & 3.0550 & 2.6770 & 2.1220 & 1.6465 \\
.15 & 3.6080 & 3.0207 & 2.2880 & 1.7213 \\
.1 & 4.4500 & 3.4720 & 2.4840 & 1.8040 \\
.05 & 5.8980 & 4.0920 & 2.7160 & 1.8960 \\
\hline
\end{tabular}




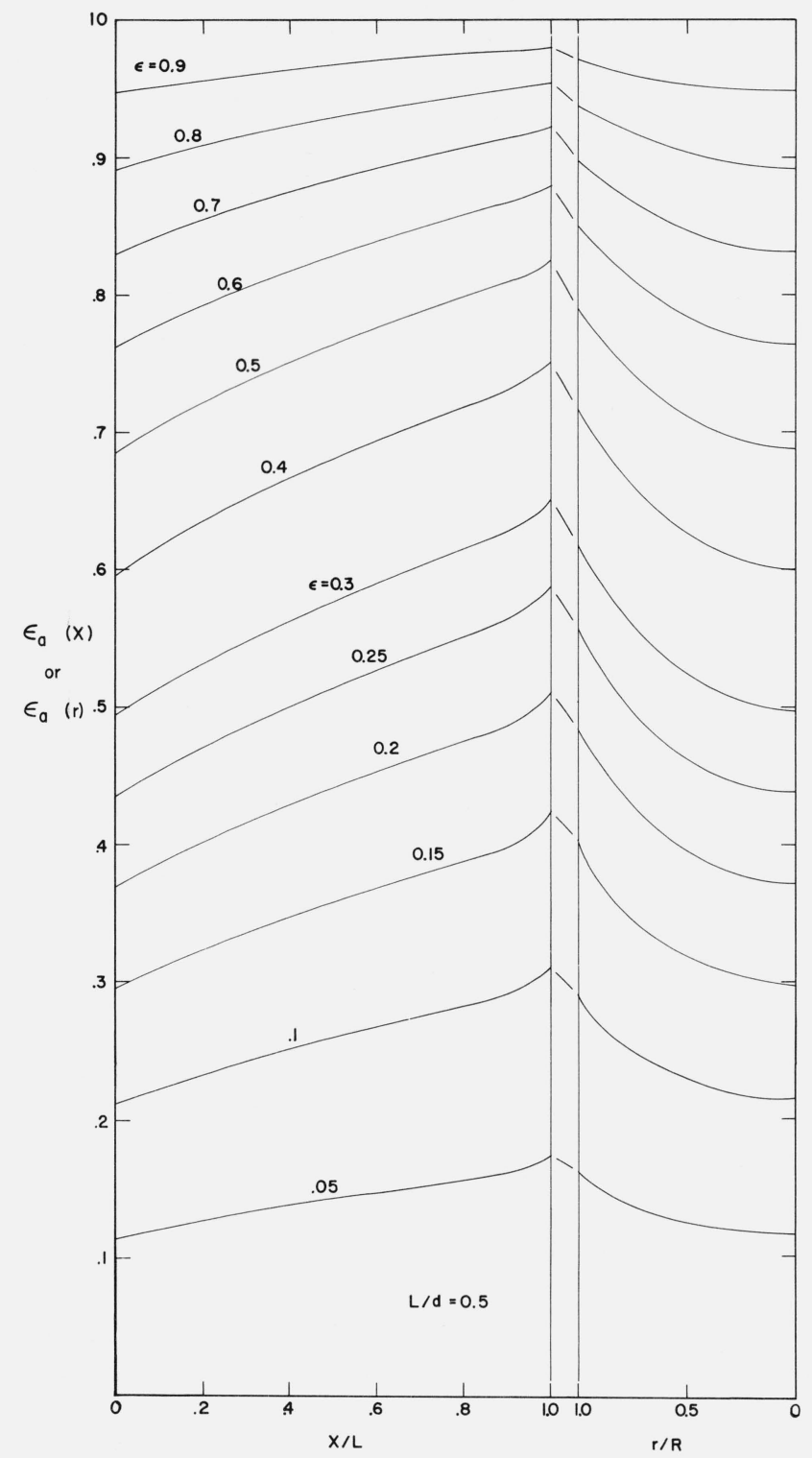

FIGURE 3. Apparent emissivity in a cylindrical cavity, $\mathrm{L} / \mathrm{d}=0.5$.

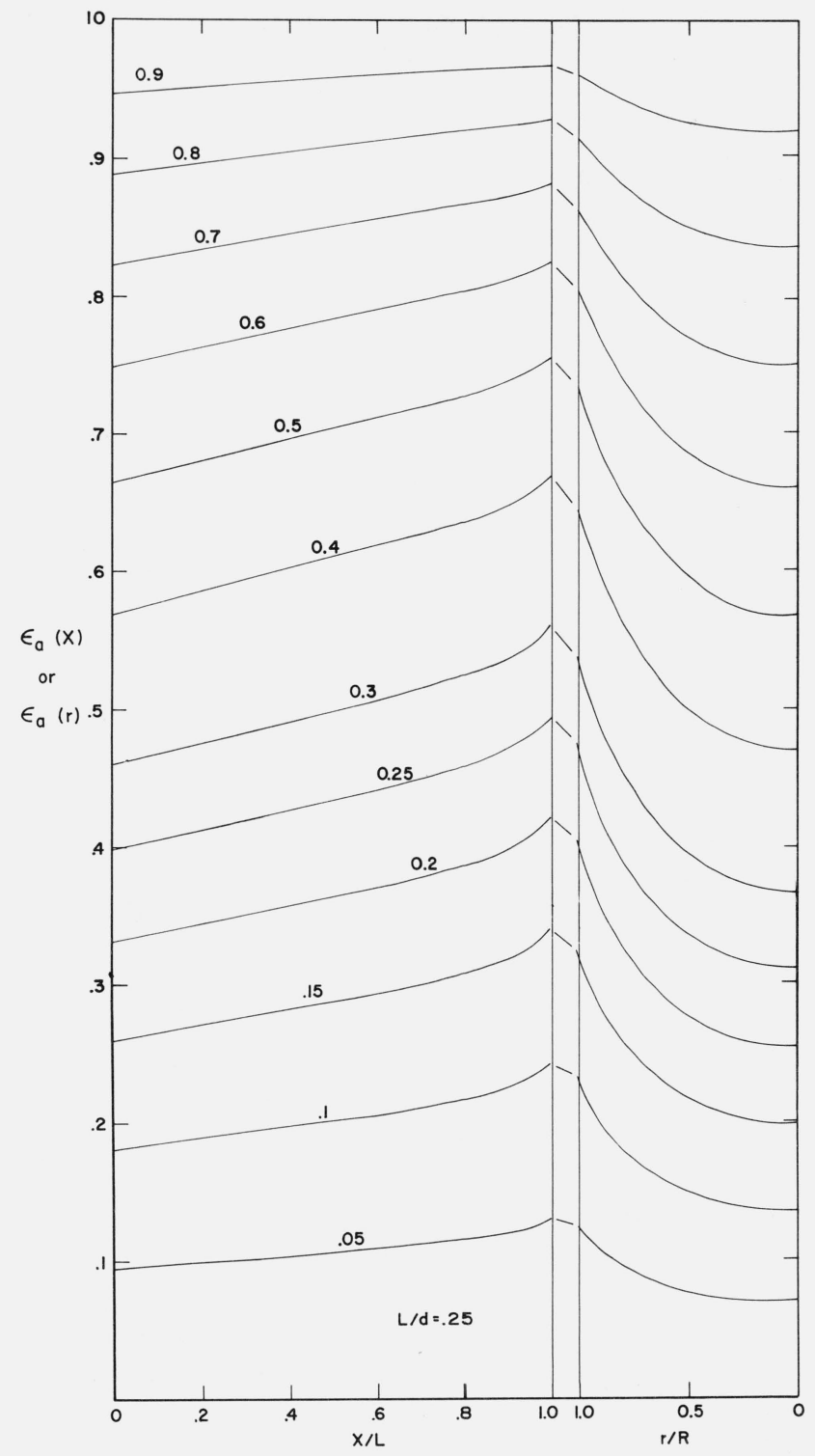

Figure 4. Apparent emissivity in a cylindrical cavity, $\mathrm{L} / \mathrm{d}=0.25$.

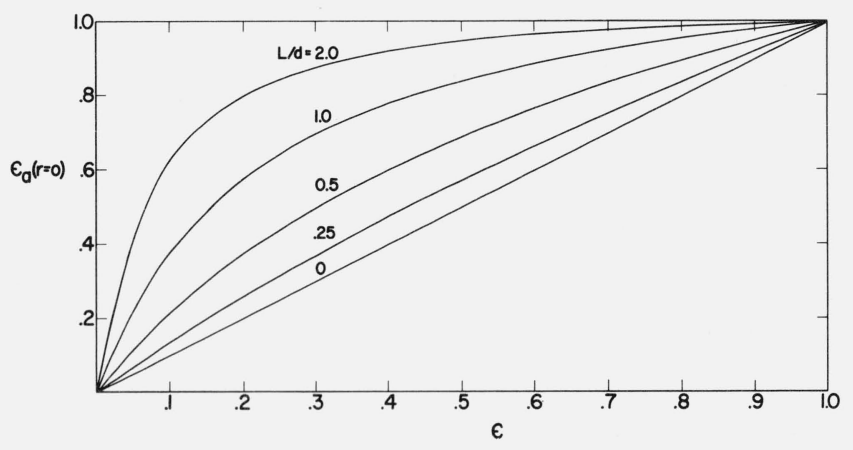

Figure 5. Apparent emissivity versus surface emissivity at the center of the bottom of cylindrical cavity. 


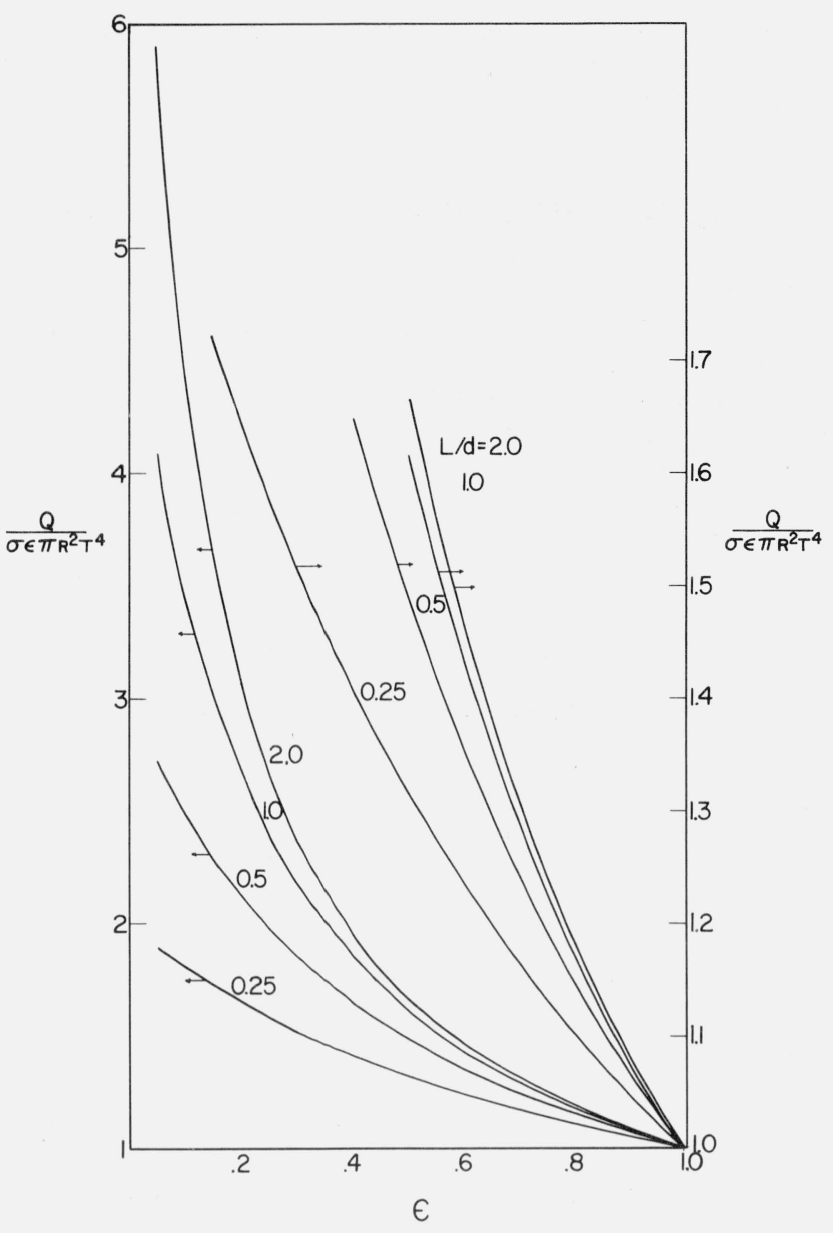

FIGURE 6. Overall heat transfer rate from cylindrical cavities versus surface emissivity.

\section{Conical Cavity}

Analytical solutions for the apparent emissivity of the surfaces of diffuse conical cavities were derived by Sparrow and Jonsson [2]. Here, the problem is to evaluate an integral of the form

$$
\frac{\cos ^{2}(\theta / 2)}{2 y \sin (\theta / 2)} \int_{0}^{1} \epsilon_{a}(t) K_{3}(y, t) d t
$$

where

$$
K_{3}(y, t)=1-|t-y| \frac{\left[(t-y)^{2}+6 t y \sin ^{2}(\theta / 2)\right]}{\left[(t-y)^{2}+4 t y \sin ^{2}(\theta / 2)\right]^{3 / 2}}
$$

and $y$ is the ratio of a surface position measured from the apex to the total length of a cone. The kernel function $K_{3}(y, t)$ has a discontinuous derivative at $y=t$ and a problem arises for the case $y=0$, which by visual inspection gives an indeterminant form. This suggests a substitution of the form

$$
\epsilon_{a l}(t)=\epsilon_{a}(y)+\gamma(t) \text {. }
$$

Letting

$$
I_{4}(y)=\frac{\cos ^{2}(\theta / 2)}{2 y \sin (\theta / 2)}\left[\int_{0}^{y} K_{3}(y, t) d t+\int_{y}^{1} K_{3}(y, t) d t\right]
$$

and performing the indicated integration, we obtain

$$
\begin{aligned}
I_{4}(y)=\frac{2 \sin (\theta / 2)-1}{2 \sin (\theta / 2)}+\frac{\cos ^{2}(\theta / 2)}{2 y \sin (\theta / 2)} \\
\quad\left[1-\frac{(1-y)^{2}+y(1+y) \tan ^{2}(\theta / 2)}{\left.\left\{(1-y)^{2}+4 y \sin ^{2}(\theta /)^{2}\right)\right\}^{1 / 2}}\right] .
\end{aligned}
$$

For the case $y=0$, the second term is an indeterminant, but can be evaluated by L'Hospital's rule. This yields

$$
I_{4}(0)=1-\sin ^{3}(\theta / 2) .
$$

The integral (9) then becomes

$$
\epsilon_{a}(y) I_{4}(y)+\frac{\cos ^{2}(\theta / 2)}{2 y \sin (\theta / 2)} \int_{0}^{1} \gamma(t) K_{3}(y, t) d t
$$

where the second term still contains the indeterminant form for $y=0$. By letting $t>0$, and applying L'Hospital's rule, we find that the integrand goes to zero. Also, $\gamma(0)=0$, so that the second term goes to zero for $y=0$, and the limit becomes

$$
\operatorname{Lim}_{y \rightarrow 0} \frac{\cos ^{2}(\theta / 2)}{2 y \sin (\theta / 2)} \int_{0}^{1} \epsilon_{a}(t) K_{3}(y, t) d t=\epsilon_{a}(0)\left[1-\sin ^{3}(\theta / 2)\right] .
$$

This is the same result derived by C. H. Page in an internal NBS Report (1952), where it was intuitively assumed that the apparent emissivity at the apex of the cone would be independent of the length of the cone, or that the cone appears infinite. The upper limit of (9) was changed to infinity; this yields $\epsilon_{l}(t)$ constant, hence equal to $\epsilon_{t}(0)$. Another way to arrive at this limit for the case $y=0$ is to assume $\gamma(t)=A_{1} t$ $+A_{2} t^{2}+\ldots$ in eq (13) and perform the indicated integration and limiting process which will give the same result as eq (14).

By making the substitution $y=x / L$ in eq (5) of [2], the equation for the apparent emissivity of a cavity maintained at uniform temperature is

$$
\begin{aligned}
\epsilon_{l l}(y)=\epsilon+(1-\epsilon) & \left\{\epsilon_{l l}(y) I_{4}(y)\right. \\
& \left.+\frac{\cos ^{2}(\theta / 2)}{2 y \sin (\theta / 2)} \int_{0}^{1} \gamma(t) K_{3}(y, t) d t\right\}
\end{aligned}
$$

which has a unique solution at $y=0$,

$$
\epsilon_{l}(0)=\frac{\epsilon}{\epsilon+(1-\epsilon) \sin ^{3}(\theta / 2)} .
$$

This is the expression for conical cavities shown by F. J. Kelly, in a paper soon to be published, as derivable from the expression for cavities used by Gouffé. It 
is also the expression derived by C. H. Page by another method (see discussion following eq (14)). Comparison with graphical quantities given in [2] for $\epsilon\left[1-\epsilon_{a}(0)\right] /(1-\epsilon)$ show almost identical agreement.

As in [2], numerical solutions were obtained by the process of iteration, where initial values of $\epsilon_{11}(y)$ were computed from

$$
\epsilon_{l 1}(y)_{1}=\frac{\epsilon}{1-(1-\epsilon) I_{4}(y)} .
$$

Because graphical presentations have appeared in [2], numerical values are not shown in this paper.

Equation (17) can be considered a good approximation to eq (15) for all cavities with surface emissivities equal to or greater than 0.7 and for all cavities with apex angles greater than $120^{\circ}$ and emissivity greater than 0.3. For emissivities and apex angles below the values cited above, the deviation between the two equations is not acceptable. An example of the percentage deviation

$$
\gamma(y)=\frac{100\left[\epsilon_{a}(y)-\epsilon_{a}(y)_{1}\right]}{\epsilon_{a}(y)}
$$

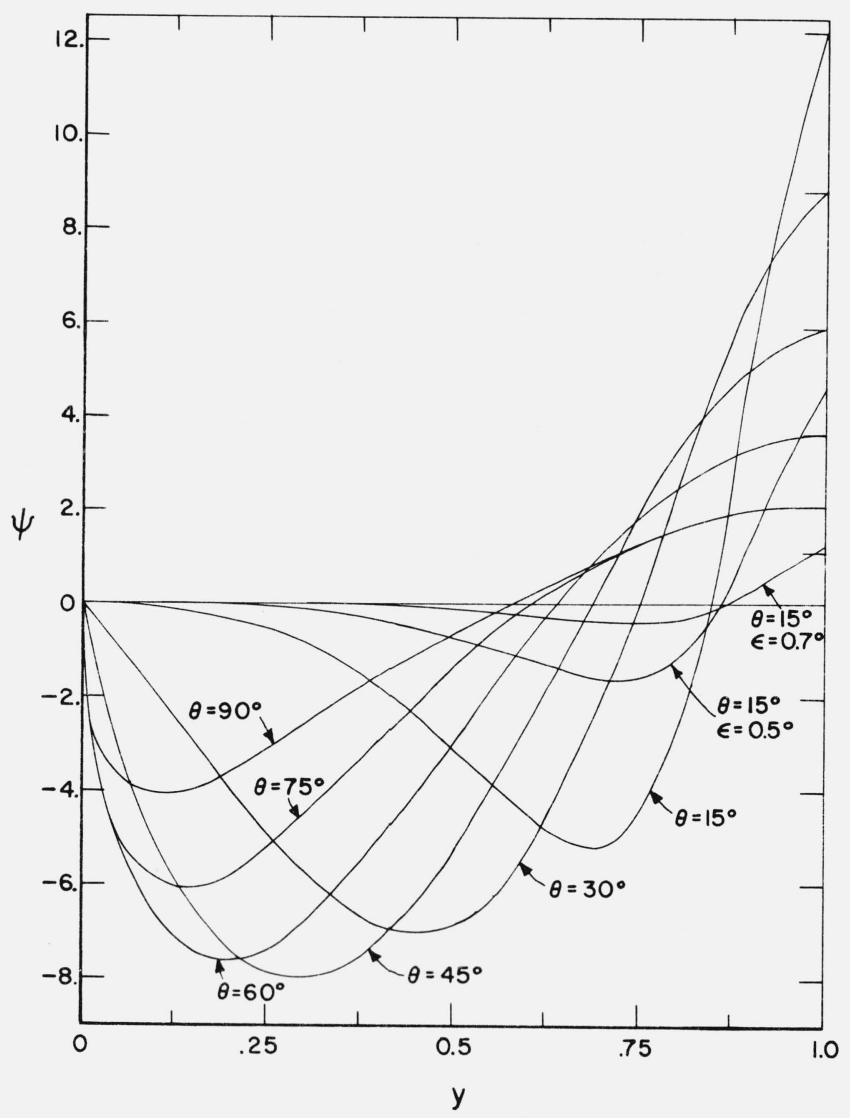

Figure 7. Percentage deviation of eq (17) from eq (1.5) versus position from apex of a conical cavity. Unless wherwise noted. surface emissivity. $\epsilon=0.3$. for various apex angles is given in figure 7 , where the average deviation over the length is -2.3 percent for $\epsilon=0.3$ and $\theta=45^{\circ}$.

\section{Cavities With Nonisothermal Surfaces}

Equations 1, 2, and 15, are concerned with cavities for which the surfaces were assumed isothermal. In the succeeding paragraphs, there are presented systems for determining the apparent emissivities of cylindrical and conical cavities with arbitrary variations of surface temperature in respect to dimensions of length and radius, assuming the surface emissivity is constant and not a function of temperature.

\subsection{Cylindrical Cavity}

For the cylindrical cavity, the integral equations take the form

$$
\begin{gathered}
\epsilon_{a}\left(x_{0}\right)=\frac{B\left(x_{0}\right)}{\sigma T_{0}^{4}}=\epsilon f\left(x_{0}\right)+(1-\epsilon) G_{1}\left[\epsilon_{a}(x), \epsilon_{a}(r)\right] \\
\epsilon_{a}(r)=\frac{B(r)}{\sigma T_{0}^{4}}=\epsilon g(r)+(1-\epsilon) G_{2}\left[\epsilon_{a}(x)\right]
\end{gathered}
$$

where

$$
\begin{aligned}
T^{4}\left(x_{0}\right) & =T_{0}^{4} f\left(x_{0}\right) ; f\left(x_{0}\right)=C_{0}+C_{1} y+\ldots+C_{n} y^{m} \\
T^{4}(r) & =T_{0}^{4} g(r) ; g(r)=1+D_{1} r^{2}+\ldots+D_{k} r^{2 k},
\end{aligned}
$$

$y=x_{0} /(L / d), T_{0}$ is an arbitrary temperature which is taken to be the temperature at $r=0$, and $G_{1}$ and $G_{2}$ are the coefficients of $(1-\epsilon)$ in (1a) and $(2 a)$, respectively. Two sets of integral equations are defined by

$$
\begin{aligned}
E_{n}\left(x_{0}\right) & =\epsilon y^{n}+(1-\epsilon) G_{1}\left[E_{n}(x), E_{n}(r)\right] \\
E_{n}(r) & =\epsilon+(1-\epsilon) G_{2}\left[E_{n}(x)\right] \\
n & =0,1,2, \ldots . m
\end{aligned}
$$

and

$$
\begin{aligned}
M_{j}\left(x_{0}\right) & =\epsilon+(1-\epsilon) G_{1}\left[M_{j}(x), M_{j}(r)\right] \\
M_{j}(r) & =\epsilon \gamma^{2 j}+(1-\epsilon) G_{2}\left[M_{j}(x)\right] \\
j & =1,2, \ldots .
\end{aligned}
$$

The apparent emissivity is then found from the following:

$$
\begin{array}{r}
\epsilon_{a l}\left(x_{0}\right)=C_{0}^{\prime} E_{0}\left(x_{0}\right)+C_{1} E_{1}\left(x_{0}\right)+\ldots+C_{m} E_{m}\left(x_{0}\right) \\
+D_{1} M_{1}\left(x_{0}\right)+\ldots .+D_{k} M_{k}\left(x_{0}\right) \\
\epsilon_{a l}(r)=C_{0}^{\prime} E_{0}(r)+C_{1} E_{1}(r)+\ldots .+C_{m} E_{m}(r) \\
+D_{1} M_{1}(r)+\ldots+D_{k} M_{k}(r)
\end{array}
$$




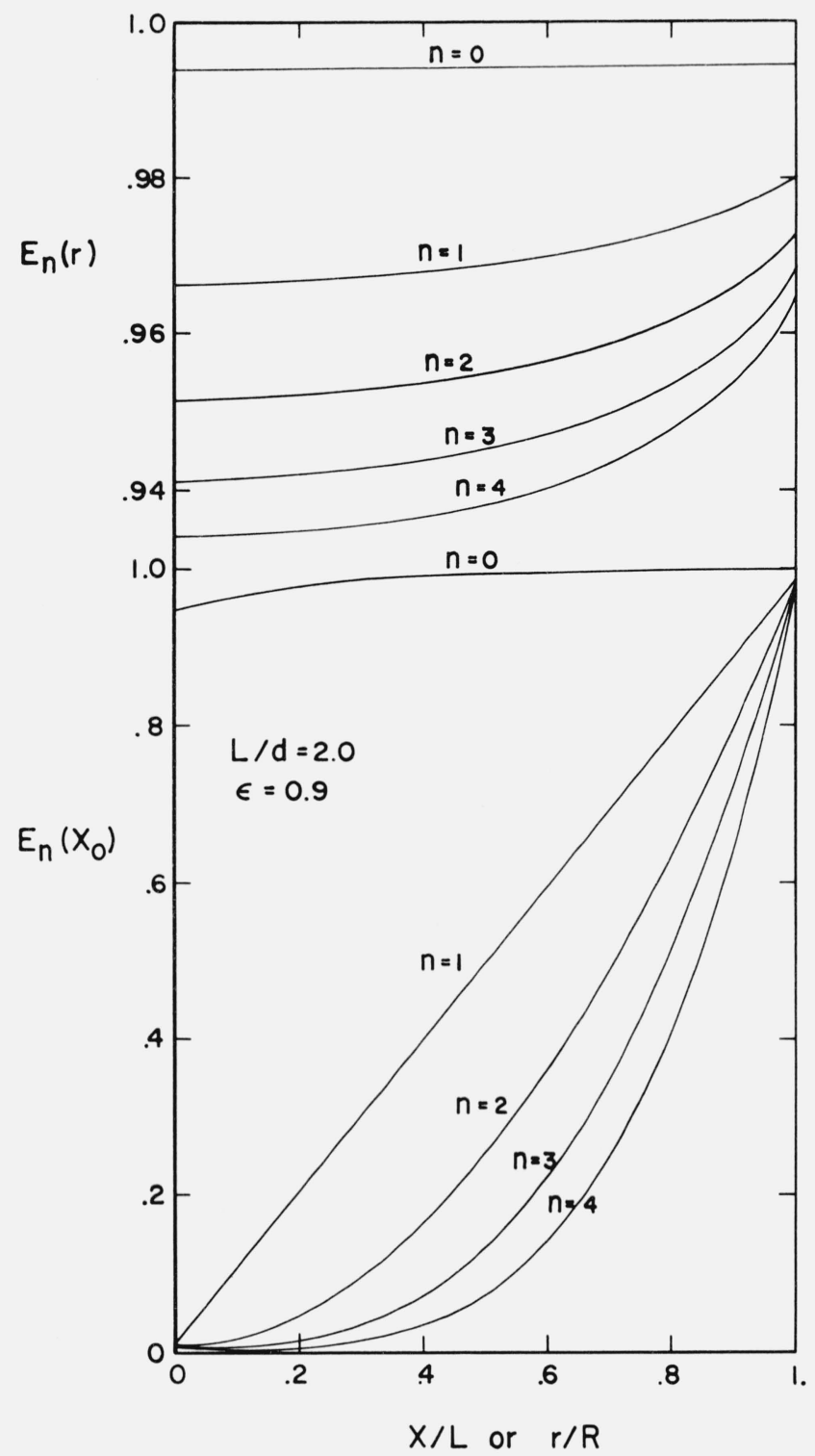

FIGURE 8. Component apparent emissivity values versus position in cylindrical cavity $(\mathrm{L} / \mathrm{d}=2.0)$ for a surface emissivity $=0.9$.

which satisfies (19) and (20) for $C_{0}^{\prime}=C_{0}-D_{1}-D_{2}-\ldots$ $D_{k}$, which gives continuity to the temperature at $x_{0}=L / d$ and $r=1$. Figures 8 and 9 show solutions of (23) and (24) for $\epsilon=0.9$ and 0.5 , respectively, with $L / d=2.0$, and $n=0,1,2,3$, and 4 .

One simplifying procedure is to assume that the bottom of the cavity is isothermal at temperature $T_{0}$. This eliminates the numerical solutions of (25) and (26); and (27) and (28) become

$$
\begin{gathered}
\epsilon_{n !}\left(x_{0}\right)=\sum_{n=0}^{m} C_{n} E_{n}\left(x_{0}\right) \\
\epsilon_{n}(r)=\sum_{n=0}^{m} C_{n} E_{n}(r) .
\end{gathered}
$$

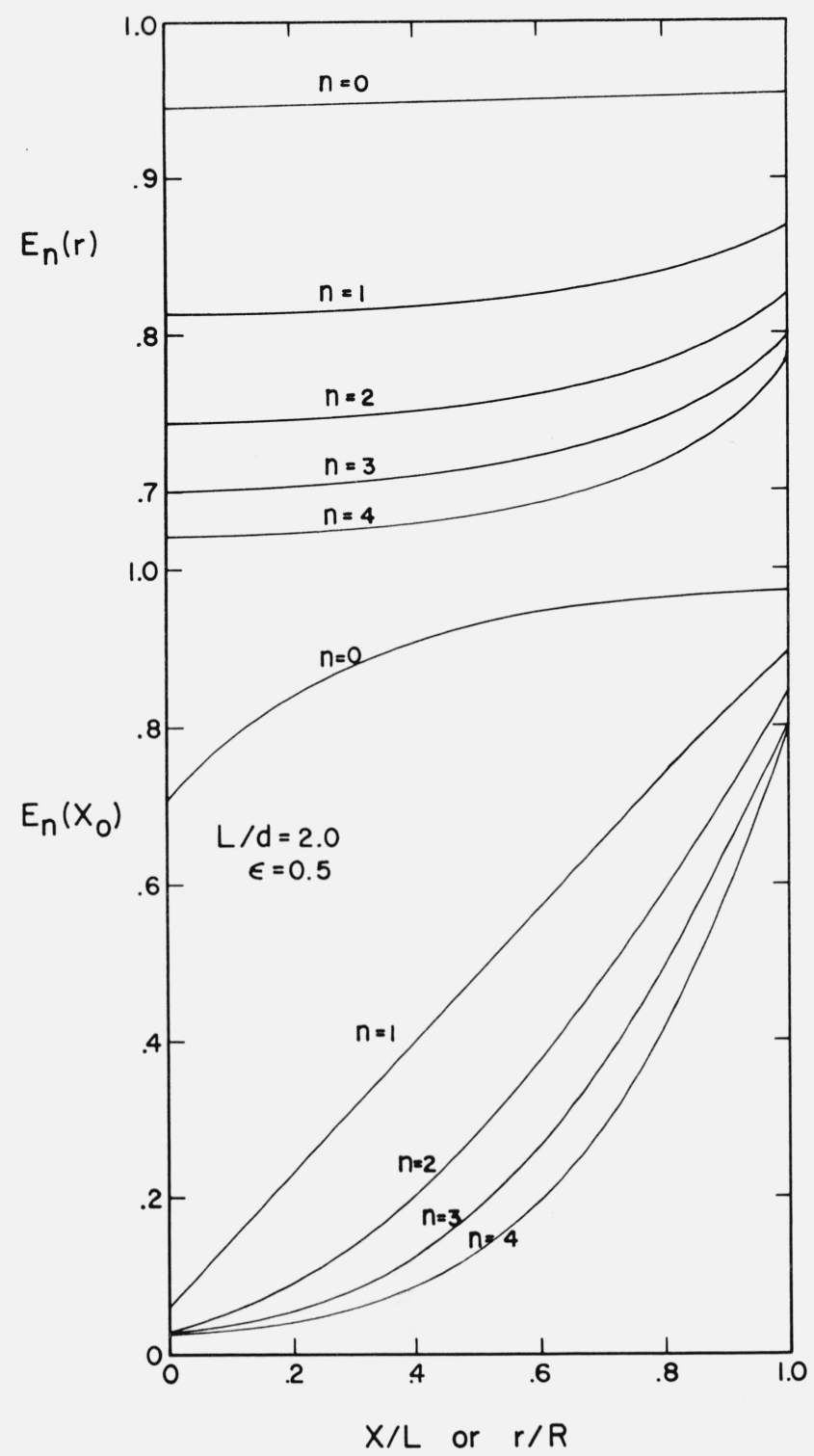

Figure 9. Component apparent emissivity values versus position in cylindrical cavity $(\mathrm{L} / \mathrm{d}=2.0)$ for a surface emissivity $=0.5$.

The ratio of the cavity radiant heat exchange $Q$ with the environment through the opening to that of a black disk of equal area at temperature $T_{0}$ is

$$
\begin{aligned}
\frac{Q}{\sigma T_{0}^{4} \pi R^{2}}=\frac{\epsilon}{(1-\epsilon)} & {\left[4 \frac{L}{d} \int_{0}^{1}\left\{f\left(x_{0}\right)-\epsilon_{l}\left(x_{0}\right)\right\} d x\right.} \\
& \left.+2 \int_{0}^{1}\left\{1-\epsilon_{a}(r)\right\} r d r\right]=\sum_{n=0}^{m} C_{n} Q_{n}
\end{aligned}
$$

where

$Q_{n}=\frac{\epsilon}{1-\epsilon}\left[4 \frac{L}{d} \int_{0}^{1}\left\{x_{0}^{n}-E_{n}\left(x_{0}\right)\right\} d x+2 \int_{0}^{0}\left\{1-E_{n}(r)\right\} r d r\right]$. 
TABLE 2. Component thermal characteristics for a cylindrical cavity, $\mathrm{L} / \mathrm{d}=0.5$, with a nonisothermal wall

\begin{tabular}{|c|c|c|c|c|c|c|}
\hline & $\epsilon=$ & 0.5 & 0.6 & 0.7 & 0.8 & 0.9 \\
\hline$E_{n}(r=0)$ & $n=0 \begin{array}{r}0 \\
1 \\
2 \\
3 \\
4\end{array}$ & $\begin{array}{r}0.68786 \\
.59722 \\
.56747 \\
.55334 \\
.54539\end{array}$ & $\begin{array}{r}0.76398 \\
.68197 \\
.65522 \\
.64260 \\
.63554\end{array}$ & $\begin{array}{r}0.83139 \\
.76344 \\
.74133 \\
.73095 \\
.72516\end{array}$ & $\begin{array}{r}0.89236 \\
.84298 \\
.82695 \\
.81946 \\
.81530\end{array}$ & $\begin{array}{r}0.94823 \\
.92158 \\
.91295 \\
.90893 \\
.90670\end{array}$ \\
\hline$E_{n}(r=1)$ & $\begin{array}{r}n=0 \\
1 \\
2 \\
3 \\
4\end{array}$ & $\begin{array}{l}.79159 \\
.74185 \\
.72380 \\
.71433 \\
.70850\end{array}$ & $\begin{array}{l}.85038 \\
.80906 \\
.79416 \\
.78637 \\
.78158\end{array}$ & $\begin{array}{l}.89769 \\
.86620 \\
.85485 \\
.84894 \\
.84530\end{array}$ & $\begin{array}{l}.93707 \\
.91592 \\
.90831 \\
.90436 \\
.90193\end{array}$ & $\begin{array}{l}.97066 \\
.96006 \\
.95627 \\
.95421 \\
.95310\end{array}$ \\
\hline$E_{n}\left(x_{0}=0\right)$ & $n=0 \begin{array}{r}0 \\
1 \\
2 \\
3 \\
4\end{array}$ & $\begin{array}{l}.68571 \\
.11781 \\
.09700 \\
.08713 \\
.08143\end{array}$ & $\begin{array}{l}.76237 \\
.10232 \\
.08423 \\
.07574 \\
.07087\end{array}$ & $\begin{array}{l}.83139 \\
.08175 \\
.06729 \\
.06057 \\
.05674\end{array}$ & $\begin{array}{l}.89188 \\
.05731 \\
.04718 \\
.04252 \\
.03988\end{array}$ & $\begin{array}{l}.94810 \\
.02986 \\
.02459 \\
.02219 \\
.02084\end{array}$ \\
\hline$Q_{n}$ & $\begin{array}{r}n=0 \\
1 \\
2 \\
3 \\
4\end{array}$ & $\begin{array}{l}.7422 \\
.4802 \\
.4006 \\
.3630 \\
.3412\end{array}$ & $\begin{array}{l}.8104 \\
.5223 \\
.4362 \\
.3959 \\
.3728\end{array}$ & $\begin{array}{l}.8687 \\
.5576 \\
.4662 \\
.4240 \\
.3999\end{array}$ & $\begin{array}{l}.9186 \\
.5876 \\
.4921 \\
.4484 \\
.4238\end{array}$ & $\begin{array}{l}.9619 \\
.6136 \\
.5149 \\
.4703 \\
.4453\end{array}$ \\
\hline
\end{tabular}

TABLE 3. Component thermal characteristics for a cylindrical cavity, $\mathrm{L} / \mathrm{d}=1$, with a nonisothermal wall

\begin{tabular}{|c|c|c|c|c|c|c|}
\hline & $\epsilon=$ & 0.5 & 0.6 & 0.7 & 0.8 & 0.9 \\
\hline$E_{n}(r=0)$ & $n=\begin{array}{r}0 \\
1 \\
2 \\
3 \\
4\end{array}$ & $\begin{array}{r}0.83957 \\
.70223 \\
.64592 \\
.61520 \\
.59658\end{array}$ & $\begin{array}{r}0.88625 \\
.76827 \\
.71951 \\
.69291 \\
.67656\end{array}$ & $\begin{array}{r}0.92309 \\
.82950 \\
.79050 \\
.76914 \\
.75593\end{array}$ & $\begin{array}{r}0.95324 \\
.88782 \\
.86032 \\
.84521 \\
.83583\end{array}$ & $\begin{array}{r}0.97847 \\
.94438 \\
.92993 \\
.92196 \\
.91700\end{array}$ \\
\hline$E_{n}(r=1)$ & $n=\begin{array}{r}0 \\
1 \\
2 \\
3 \\
4\end{array}$ & $\begin{array}{l}.87784 \\
.79987 \\
.76831 \\
.75077 \\
.73989\end{array}$ & $\begin{array}{l}.91531 \\
.85305 \\
.82782 \\
.81384 \\
.80500\end{array}$ & $\begin{array}{l}.94353 \\
.89741 \\
.87869 \\
.86831 \\
.86170\end{array}$ & $\begin{array}{l}.96591 \\
.93563 \\
.92333 \\
.91651 \\
.91216\end{array}$ & $\begin{array}{l}.98431 \\
.96942 \\
.96338 \\
.96003 \\
.95789\end{array}$ \\
\hline$E_{n}\left(x_{0}=0\right)$ & $n=\begin{array}{r}0 \\
1 \\
2 \\
3 \\
4\end{array}$ & $\begin{array}{l}.70148 \\
.09719 \\
.06924 \\
.05704 \\
.05059\end{array}$ & $\begin{array}{l}.77174 \\
.08009 \\
.05627 \\
.04613 \\
.04075\end{array}$ & $\begin{array}{l}.83533 \\
.06114 \\
.04237 \\
.03455 \\
.03043\end{array}$ & $\begin{array}{l}.89394 \\
.04117 \\
.02816 \\
.02284 \\
.02007\end{array}$ & $\begin{array}{l}.94858 \\
.02068 \\
.01396 \\
.01127 \\
.00988\end{array}$ \\
\hline$Q_{n}$ & $n=\begin{array}{r}0 \\
1 \\
2 \\
3 \\
4\end{array}$ & $\begin{array}{l}.8078 \\
.4088 \\
.3018 \\
.2552 \\
.2281\end{array}$ & $\begin{array}{l}.8598 \\
.4235 \\
.3100 \\
.2614 \\
.2343\end{array}$ & $\begin{array}{l}.9036 \\
.4334 \\
.3149 \\
.2651 \\
.2382\end{array}$ & $\begin{array}{l}.9404 \\
.4401 \\
.3176 \\
.2671 \\
.2405\end{array}$ & $\begin{array}{l}.9721 \\
.4447 \\
.3189 \\
.2682 \\
.2419\end{array}$ \\
\hline
\end{tabular}

TABLE 4. Component thermal characteristics for a cylindrical cavity, $\mathrm{L} / \mathrm{d}=2$, with a nonisothermal wall

\begin{tabular}{|c|c|c|c|c|c|c|}
\hline & $\epsilon=$ & 0.5 & 0.6 & 0.7 & 0.8 & 0.9 \\
\hline$E_{n}(r=0)$ & $n=0 \begin{array}{r}0 \\
1 \\
2 \\
3 \\
4\end{array}$ & $\begin{array}{r}0.94601 \\
.81188 \\
.74333 \\
.70026 \\
.67076\end{array}$ & $\begin{array}{r}0.96371 \\
.85575 \\
.79923 \\
.76313 \\
.73806\end{array}$ & $\begin{array}{r}0.97639 \\
.89514 \\
.85163 \\
.82354 \\
.80386\end{array}$ & $\begin{array}{r}0.98599 \\
.93171 \\
.90204 \\
.88269 \\
.86902\end{array}$ & $\begin{array}{r}0.99364 \\
.96644 \\
.95130 \\
.94132 \\
.93423\end{array}$ \\
\hline$E_{n}(r=1)$ & $\begin{array}{r}n=0 \\
1 \\
2 \\
3 \\
4\end{array}$ & $\begin{array}{l}.95338 \\
.86738 \\
.82589 \\
.80054 \\
.78341\end{array}$ & $\begin{array}{l}.96864 \\
.90331 \\
.87139 \\
.85166 \\
.83819\end{array}$ & $\begin{array}{l}.97949 \\
.93281 \\
.90974 \\
.89543 \\
.88559\end{array}$ & $\begin{array}{l}.98773 \\
.95796 \\
.94312 \\
.93388 \\
.92750\end{array}$ & $\begin{array}{l}.99436 \\
.98006 \\
.97288 \\
.96840 \\
.96530\end{array}$ \\
\hline$E_{n}\left(x_{0}=0\right)$ & $n=0 \begin{array}{r}0 \\
1 \\
2 \\
3 \\
4\end{array}$ & $\begin{array}{l}.70647 \\
.06475 \\
.03595 \\
.02561 \\
.02066\end{array}$ & $\begin{array}{l}.77428 \\
.05108 \\
.02713 \\
.01890 \\
.01500\end{array}$ & $\begin{array}{l}.83653 \\
.03755 \\
.01920 \\
.01309 \\
.01034\end{array}$ & $\begin{array}{l}.89437 \\
.02455 \\
.01209 \\
.00809 \\
.00633\end{array}$ & $\begin{array}{l}.94868 \\
.01204 \\
.00573 \\
.00376 \\
.00292\end{array}$ \\
\hline$Q_{n}$ & $\begin{array}{r}n=0 \\
1 \\
2 \\
3 \\
4\end{array}$ & $\begin{array}{l}.8331 \\
.2884 \\
.1737 \\
.1309 \\
.1087\end{array}$ & $\begin{array}{l}.8772 \\
.2863 \\
.1679 \\
.1258 \\
.1051\end{array}$ & $\begin{array}{l}.9142 \\
.2835 \\
.1628 \\
.1210 \\
.1012\end{array}$ & $\begin{array}{l}.9463 \\
.2801 \\
.1580 \\
.1167 \\
.0977\end{array}$ & $\begin{array}{l}.9746 \\
.2766 \\
.1534 \\
.1130 \\
.0948\end{array}$ \\
\hline
\end{tabular}

Tables 2, 3, and 4 give values of the component thermal radiation characteristics $E_{n}(r=0), E_{n}(r=1)$, $E_{n}\left(x_{0}=0\right)$ and $Q_{n}$ for $L / d=2$. 1. and $0.5, \epsilon=0.5,0.6$, $0.7,0.8$, and 0.9 , and $n=0,1,2,3$, and 4 . For the sake of a numerical example, assume a cylindrical cavity, $\epsilon=0.7$, where the temperature decreases linearly from a temperature $T_{0}$ at its base to a value $0.9 T_{0}$ at the open end, or, from $(21), f(x)=0.6561+$ $0.2916 y+0.0486 y^{2}+0.0036 y^{3}+0.0001 y^{4}$. For $L / d=2$, from eq $(31)$ and table 4 , we find

$$
\begin{aligned}
\frac{Q}{\sigma T_{0}^{4} \pi R^{2}}= & 0.6561 \times 0.9142+ \\
+ & 0.2916 \times 0.2835 \\
+0.048 \times 0.1628+ & 0.0036 \times 0.1210 \\
& +0.0001 \times 0.1012=0.6908
\end{aligned}
$$

which is compared to a value of 0.9142 for the isothermal cavity at $T_{0}$.

\subsection{Conical Cavity}

For the conical cavity, the integral equation takes the form

$$
\epsilon_{a}(y)=\frac{B(y)}{\sigma T_{0}}=\epsilon f(y)+(1-\epsilon) G_{3}\left[\epsilon_{a}(t)\right]
$$

where

$$
T^{4}(y)=T_{0}^{4} f(y)=1+C_{1} y+C_{2} y^{2}+\ldots+C_{m} y^{m}
$$

and $T_{0}$ is the temperature at the apex of the cone, $G_{3}\left[\epsilon_{a}(t)\right]$ is the coefficient of $(1-\epsilon)$ in (15) and $B(y)$ is the radiant emission.

A set of $m$ integral equations is defined by

$$
\begin{aligned}
E_{n}(y) & =\epsilon y^{n}+(1-\epsilon) G_{3}\left[\epsilon_{a}(t)\right] \\
n & =0,1,2, \ldots, m .
\end{aligned}
$$

Then the apparent emissivity is

$$
\epsilon_{a}(y)=E_{0}(y)+C_{1} E_{1}(y)+\ldots+C_{m} E_{m}(y) .
$$

Evaluation of (34) for substitution in (35) gives an exact solution to (32). An approximation from eq (17) useful within the limits cited in section 3 is

$$
\boldsymbol{\epsilon}_{a}(y)=\frac{\boldsymbol{\epsilon} f(y)}{1-(1-\boldsymbol{\epsilon}) I_{4}(y)} .
$$

\section{Discussion}

Sections 2 and 3 present an analytical treatment by appropriate substitution in integrals which exhibit slope discontinuities or apparent discontinuities or both at critical points, such as the corner of a cylin- 
drical cavity and the apex of a conical cavity. The substitutions (3), (4), (7), and (10) make the appropriate integrands go to zero when evaluated at the critical points and points of slope discontinuity, whereby the awkward behavior of the integrand is practically eliminated. With the transformed problem, as given by (1a), (2a), and (15), accurate numerical results are readily obtained by use of a sufficiently small integration step and the fulfillment of the convergence criterion for the successive iterations.

It may be remarked that the very good agreement found between the numerical results arrived at by extrapolation in references 1,2 , and 3 , and the rigorous analytical formulation given here shows that any error introduced by linear extrapolation was negligible. However, to avoid the uncertainties involved in extrapolation, this paper does provide the equations (isothermal and nonisothermal) in a direct form for computation. The suggested method is equally applicable in other cases of integral equations in which similar discontinuities are encountered.

The nonisothermal cylindrical cavity has been treated by Sparrow [5]. For the same values of the parameters, there is generally good agreement between the numerical results presented in this paper and reference 5. Two assumptions were made in the treatment [5]; namely, (1) the temperature over the base of the cavity was isothermal, and (2) the apparent emissivity over the base was constant. For relatively deep cavities, these assumptions can be considered reasonable, but for shallow cavities there is some question as to the validity of these assumptions. Figures 2,3 , and 4 , for example, show a considerable variation of apparent emissivity $\epsilon_{\ell}(r)$ over the base of the cavity.

Although numerical results are not presented in this paper for temperature variations over the base of the cavity, this can be accomplished by evaluation of eqs (25) and (26). Also, numerical results are presented for general linear temperature distributions, but may be evaluated for all temperature distributions that may be represented in a polynomial or transcendental form.

\section{References}

[1] E. M. Sparrow, L. U. Albers, and E. R. G. Eckert, Thermal radiation characteristics of cylindrical enclosures, J. Heat Transfer, Trans. ASME, Series C 84, 73-81 (1962).

[2] E. M. Sparrow and V. K. Jonsson, Radiant emission characteristics of diffuse conical cavities. J. Opt. Soc. Am. 53, 816-821 (1963).

[3] S. H. Lin, Radiant Interchange in Cavities and Passages with Specularly and Diffusely Reflecting Surfaces, $\mathrm{Ph}$. D. Thesis, Department of Mechanical Engineering, University of Minnesota, Minneapolis, Minn., March 1964.

[4] F. J. Kelly and D. G. Moore, A test of analytical expressions for the thermal emissivity of shallow cylindrical cavities, Appl. Opt. 4, 31 (1965).

[5] E. M. Sparrow, Radiant emission characteristics of nonisothermal cylindrical cavities, Appl. Opt. 4, 4l-43 (1965).

(Paper 70C2-224) 